\title{
Entornos híbridos de enseñanza y aprendizaje para promover la personalización del aprendizaje
}

\section{(Hybrid Teaching and Learning Environments to Promote Personalized Learning)}

\author{
Anna Engel \\ César Coll \\ Universitat de Barcelona (España)
}

DOI: https://doi.org/10.5944/ried.25.1.31489

\section{Cómo referenciar este artículo:}

Engel, A., y Coll, C. (2022). Entornos híbridos de enseñanza y aprendizaje para promover la personalización del aprendizaje. RIED. Revista Iberoamericana de Educación a Distancia, 25(1), pp. 225-242. https://doi.org/10.5944/ ried.25.1.31489

\section{Resumen}

El artículo analiza la potencialidad de los entornos híbridos para personalizar el aprendizaje escolar tomando como punto de partida algunas características de los centros educativos que han logrado afrontar las dificultades provocadas por el impacto de la COVID-19 con mayor rapidez, facilidad y eficacia. Dos características son objeto de especial atención. La primera es la existencia de un planteamiento educativo presidido por la idea de personalización del aprendizaje, entendida como un conjunto de estrategias organizativas, curriculares y didácticas orientadas a promover y reforzar el sentido y el valor personal que el alumnado atribuye a los aprendizajes escolares. Y la segunda, el uso de las tecnologías digitales de la información y la comunicación para conformar entornos de aprendizaje que difuminan las fronteras entre lo presencial y lo virtual y entre lo escolar y lo no escolar y, con ello, permiten facilitar, transformar o potenciar las estrategias de personalización del aprendizaje. En este marco, se exploran las posibilidades que ofrecen los entornos híbridos para facilitar, transformar o potenciar el diseño y la puesta en práctica de diferentes estrategias de personalización del aprendizaje, así como para el acompañamiento del alumnado y la coordinación del profesorado. El trabajo concluye con una reflexión sobre los entornos personales de aprendizaje híbridos como un marco idóneo para la implementación de estrategias de personalización del aprendizaje.

Palabras clave: entornos híbridos de enseñanzay aprendizaje; personalización del aprendizaje; sentido del aprendizaje; tecnologías digitales de la información y la comunicación. 


\begin{abstract}
This paper analyses the potential of hybrid environments to personalize learning, taking as a starting point some features of educational centres that have managed more quickly, easily, and effectively the difficulties caused by the impact of COVID-19. We focus on two main characteristics. First, the existence of an educational approach presided by the idea of personalization of learning, understood as a set of different organizational, curricular, and didactic strategies aimed at promoting and reinforcing the meaning that students attribute to school learning. And the second one is the use of digital information and communication technologies to shape learning environments that blur the boundaries between the faceto-face and the virtual, and between the school as well as the out-of-school. Within this framework, we explore the possibilities offered by hybrid environments for designing and implementing different strategies for personalizing learning are explored, as well as for the monitoring of students and coordination of teachers. This paper concludes with a reflection on hybrid personal learning environments as an ideal framework for the implementation of learning personalization strategies.
\end{abstract}

Keywords: hybrid teaching and learning environments; personalizing learning; sense of learning; digital information and communication technologies.

En España, como en la mayoría de los países, los efectos de la pandemia global COVID-19 en la educación empezaron con el confinamiento obligatorio de la población el 14 de marzo de 2020, que se prolongó hasta final de curso 2019-20. Durante este periodo se suspendieron las clases presenciales y se cerraron los centros educativos. Una parte importante de los centros educativos y sus equipos docentes implementaron prácticas educativas que se han caracterizado como enseñanza remota de emergencia (Hodges et al., 2020).

Durante el curso 2020-21, las restricciones derivadas de la necesidad de distanciamiento personal y de atender a los alumnos confinados en sus hogares ha obligado a los centros educativos a adoptar un amplio y diverso espectro de modelos de educación híbrida. Algunos centros han optado por un modelo en el que una parte del alumnado de los grupos clase realiza la actividad habitual presencialmente en las instalaciones escolares, mientras que el resto del alumnado se une de manera virtual a la actividad desde sus hogares. En otros centros la educación híbrida se ha entendido como una alternancia de clases presenciales en el centro y de seguimiento en línea, de modo que algunos alumnos permanecen unos días en el centro y otros en el hogar y, al cabo de un cierto periodo de tiempo, intercambian su ubicación para el desarrollo de las actividades escolares. En este caso, lo más frecuente es que las actividades presenciales sigan el formato ordinario y las actividades en línea se organicen en torno a la entrega de materiales, el trabajo autónomo del alumnado y momentos de comunicación sincrónica con el profesorado para la resolución de dudas sobre los contenidos o la realización de las tareas. 
En el último año se ha publicado un considerable número de informes y análisis sobre los efectos de la pandemia en la educación (por ejemplo, Departament d'Educació-IRE.UB, 2021; Consejo Escolar del Estado, 2021; Fundación SM, 2020; UNICEF, 2020). En conjunto, los resultados de estos informes destacan que los centros educativos se han tenido que enfrentar a numerosos problemas. Así, por ejemplo, se ha detectado que el profesorado siente que no tiene suficientes competencias digitales ni conocimientos pedagógicos para aprovechar las tecnologías digitales de la información y la comunicación (TIC, en adelante) en la docencia; la falta de competencias digitales y de autonomía del alumnado para aprender en línea; la falta de conectividad y de dispositivos tecnológicos en algunos centros y hogares; el escaso o nulo conocimiento que tienen muchos centros educativos de las situaciones familiares de una buena parte de su alumnado y su poco contacto con otros actores sociales de la comunidad. También se han detectado, sin embargo, aspectos positivos como, por ejemplo, que muchos equipos docentes se han implicado activamente en diseñar experiencias educativas mediadas por las tecnologías digitales con una visión de futuro y no solo para hacer frente a la crisis provocada por la pandemia, o también el apoyo emocional proporcionado a menudo por el profesorado a sus alumnos y sus familias.

Con todo, un buen número de centros han logrado afrontar y superar estas dificultades con una relativa facilidad y eficacia. Se trata principalmente de centros que previamente a la pandemia habían implementado ya una serie de enfoques, procedimientos y estrategias que les han permitido hacer frente a la nueva situación sin tener que improvisar totalmente las respuestas (ver, por ejemplo, INS Cardener, INS Cal Gravat o INS Quatre Cantons en Coll, 2018). En primer lugar, son centros en los que las TIC, más allá de su uso habitual como herramientas para enseñar y aprender, son utilizadas también para configurar entornos de enseñanza y aprendizaje en línea articulados e integrados con entornos presenciales. Para estos centros la adopción de un modelo de educación híbrida ha sido un paso natural, porque su profesorado y su alumnado estaban ya habituados a trabajar combinando ambos formatos de actividad conjunta. En segundo lugar, son centros que mantienen un contacto estrecho con las familias que va más allá de las reuniones colectivas, trimestrales o puntuales para resolver problemas particulares o casos conflictivos. El conocimiento de las realidades familiares ha permitido a estos centros realizar un seguimiento sistemático y un acompañamiento de los alumnos en coordinación con sus familias, así como ajustar de forma contingente las ayudas a sus necesidades y reforzar en mayor medida a los más vulnerables. En tercer lugar, y en estrecha relación con los dos aspectos mencionados, son centros que hacen habitualmente un uso pedagógico de los recursos comunitarios y de las redes de actores sociales de su entorno más próximo y no limitan el desarrollo de las actividades de enseñanza y aprendizaje al centro escolar. Ello les ha permitido aprovechar las dinámicas y los recursos comunitarios en lugar de limitarse a proyectar sobre el entorno familiar las actividades escolares. 
En suma, se trata de centros que, por una parte, han sabido generar ambientes híbridos que aprovechan las affordances de la actividad conjunta del profesorado y del alumnado en línea y cara a cara para mejorar las formas de enseñar y aprender. Por otra parte, son centros que han sido capaces de romper el tradicional encapsulamiento del sistema educativo creando estrechas sinergias con las familias y la comunidad para promover el aprendizaje del alumnado. Este alineamiento entre lo que se hace dentro y fuera de la escuela contribuye a reforzar el sentido y valor personal que el alumnado atribuye a los aprendizajes escolares y supone un importante avance hacia la personalización del aprendizaje escolar (Coll, 2016, 2018).

En lo que sigue vamos a argumentar cómo los dos aspectos señalados, el uso de las TIC para configurar entornos híbridos de enseñanza y aprendizaje y las estrategias de personalización del aprendizaje, pueden confluir y reforzarse mutuamente para generar experiencias de aprendizaje que ayuden a los alumnos a dar sentido a lo que aprenden, porque solo de este modo los aprendizajes que hagan serán realmente significativos para ellos.

\section{LA PERSONALIZACIÓN DEL APRENDIZAJE ESCOLAR}

Los enfoques de personalización del aprendizaje conforman, según Cuban (2018), un continuo. En un polo del continuo encontramos propuestas centradas en el profesor que utilizan las TIC para adaptar las actividades y contenidos de aprendizaje al desempeño individual del alumnado. En el otro polo del continuo encontramos propuestas centradas en el aprendiz, en las que las TIC se utilizan para promover el protagonismo de los alumnos en el control de sus procesos de aprendizaje atendiendo sus intereses y pasiones.

Por nuestra parte, nos situamos claramente en este segundo polo. La importancia de tener en cuenta la voz del alumno en la planificación y desarrollo de las actividades de enseñanza y aprendizaje, y el reconocimiento y aceptación de la capacidad del alumno para controlar su propio proceso de aprendizaje adquieren así una importancia crucial en las propuestas y experiencias de personalización del aprendizaje escolar (Coll, 2016). Los aprendices y los aprendizajes están en el centro del proceso, como en todas las propuestas constructivistas, y la enseñanza se entiende como una ayuda ajustada a las características y necesidades de los alumnos a lo largo de sus procesos de aprendizaje. Sin embargo, la personalización del aprendizaje supone, además, que las necesidades no solo son detectadas desde el exterior, sino que el aprendiz contribuye a identificarlas y a definir y controlar cómo satisfacerlas en función de sus intereses y opciones personales (Coll, 2018).

Una visión similar está presente en el National Education Technology Plan del Departamento de Educación de EE.UU. (2016) que caracteriza el aprendizaje personalizado como los planteamientos en los que 
los objetivos de aprendizaje, los enfoques de instrucción y el contenido de la enseñanza (y su secuencia) pueden variar según las necesidades del alumno. Además, las actividades de aprendizaje son significativas y relevantes para los alumnos, están impulsadas por sus intereses y, a menudo, son iniciadas por ellos mismos (p. 7).

En la misma línea, Bray y McClaskey (2014) afirman que "En un entorno centrado en el alumno, los alumnos tienen el control y co-diseñan su aprendizaje. El profesor es su guía en su trayectoria personal" (p. 14). Este control y responsabilidad compartidos es un movimiento estratégico para aumentar la motivación, el compromiso y la implicación del alumno (Toshalis y Nakkula, 2012) con el objetivo más amplio de enseñarle a ser un "aprendiz experto" bien preparado para toda una vida de aprendizaje (Bray y McClaskey, 2014).

Desde nuestra perspectiva, la personalización del aprendizaje es un planteamiento educativo dirigido a promover y reforzar el sentido y valor personal que los alumnos atribuyen a lo que aprenden en los centros educativos. Un aprendizaje tiene sentido y valor personal para el aprendiz cuando le ayuda a conocerse y entenderse mejor a sí mismo y al mundo que le rodea, así como a actuar en y sobre esa realidad en la que está inmerso, y a proyectarse hacia el futuro construyendo planes de acción y escenarios que le implican personalmente.

Los resultados aportados por las investigaciones sobre los procesos de aprendizaje realizadas en el transcurso de las últimas décadas (ver, por ejemplo, Dumont et al., 2010; Learning Policy Institute y Turnaround for Children, 2021; Sawyer, 2014) han permitido identificar características de las situaciones de aprendizaje más eficaces para promover en los aprendices la realización de aprendizajes con sentido $\mathrm{y}$ valor personal. Sobre esta base, se han identificado un conjunto de estrategias y actuaciones docentes entre las que destacan las siguientes (Coll, 2018, pp. 16-17):

- Tomar en consideración los intereses, objetivos y opciones del alumnado en el diseño, la planificación y desarrollo de las actividades de enseñanza y aprendizaje.

- Reconocer, aceptar y permitir ejercer la capacidad de decisión del alumnado sobre algunos o todos los componentes de las actividades de enseñanza y aprendizaje (objetivos, contenidos, materiales, apoyos, tareas, secuencia, evaluación, etc.).

- Identificar experiencias de aprendizaje significativas para el alumnado que tienen su origen fuera de la escuela y conectarlas con las actividades escolares de enseñanza y aprendizaje.

- Incorporar tiempos y espacios específicos para la revisión, reflexión y valoración, tanto a nivel individual como colectivo, de experiencias de aprendizaje significativas para el alumnado con independencia del contexto de actividad, escolar o no escolar, en que hayan tenido lugar. 
- Incorporar tiempos y espacios específicos para que los alumnos reflexionen sobre su manera de abordar las actividades y tareas de aprendizaje, escolares y no escolares, y sobre la visión que tienen de sí mismos como aprendices.

- Poner el énfasis en contenidos de aprendizaje social y culturalmente relevantes mediante la participación en actividades e iniciativas comunitarias.

- Incorporar y aprovechar los recursos y oportunidades para aprender disponibles en el entorno comunitario y en los diferentes contextos de actividad a los que el alumnado puede acceder.

- Incorporar y aprovechar los recursos y oportunidades de aprendizaje disponibles y accesibles en la red.

- Organizar la acción educativa a partir del establecimiento de Planes Personales de Aprendizaje.

- Seguir y acompañar al alumnado a partir de la coordinación de los equipos docentes.

Permítasenos destacar aún dos ideas para cerrar este apartado. La primera es que un entorno educativo personalizado pone el énfasis en la toma en consideración de la voz del alumno y el reconocimiento y aceptación de su capacidad de decisión en la dirección, control y responsabilidad de su propio proceso de aprendizaje. Sin embargo, reconocer al alumnado la capacidad de tomar decisiones no significa cederle el control y la responsabilidad total de su propio proceso de aprendizaje desde el inicio hasta el final del proceso. Antes bien, significa acompañarlo y ayudarlo para que puedan asumir progresivamente ese control y responsabilidad. En los momentos iniciales la toma de decisiones está muy controlada por el profesor y, a medida que el alumno se muestra más autónomo, los apoyos y ayudas que proporciona el profesor van retirándose progresivamente, o van siendo sustituidos por otros que suponen tipos y grados de ayuda menores, permitiendo que el alumno alcance cuotas cada vez mayores de responsabilidad y control en la toma de decisiones sobre las tareas y contenidos, y en último término sobre su propio aprendizaje. En este planteamiento el papel del profesorado y de los pares es fundamental para ayudar y orientar al alumnado a explorar, identificar y valorar sus intereses y opciones personales de modo que de forma gradual pueda asumir el control y la responsabilidad de su propio proceso de aprendizaje (Coll, 2016).

La segunda idea es que la potencialidad de una determinada estrategia de personalización de aprendizaje está condicionada por su confluencia con otras estrategias de personalización. En otras palabras, cualquiera de las estrategias mencionadas ve incrementada su efectividad cuando aparece articulada en la misma práctica educativa o espacio de aprendizaje con otras estrategias de personalización. 


\section{ESTRATEGIAS DE PERSONALIZACIÓN DEL APRENDIZAJE EN ENTORNOS HÍBRIDOS DE ENSEÑANZA Y APRENDIZAJE}

Un entorno de enseñanza y aprendizaje híbrido, mixto o mezclado implica combinar espacios de aprendizaje físicos en los que los participantes están copresentes en el mismo lugar con espacios de aprendizaje en línea o virtuales donde los participantes están ubicados en distintos lugares, así como también espacios de aprendizaje en línea con los participantes compartiendo un mismo lugar físico, eliminando las barreras entre la formación presencial y virtual (Miao et al., 2020; World Bank, 2020).

Al igual que los espacios de aprendizaje físicos, los espacios de aprendizaje en línea permiten desarrollar actividades con diferentes formas de organización social (individual, pequeño grupo o gran grupo). Sin embargo, a diferencia de estos últimos, los espacios de aprendizaje en línea permiten desarrollar actividades de forma sincrónica o asincrónica, con independencia del lugar donde se lleven a cabo, y configurar espacios de trabajo que integran distintos sistemas semióticos (letras y textos escritos, imágenes fijas o en movimiento, lenguaje oral, sonidos, datos numéricos, gráficos, etc.) y amplían hasta límites insospechados las capacidades de profesores y alumnos para (re)presentar, procesar, transmitir y compartir informaciones y conocimientos.

Se puede personalizar el aprendizaje sin necesidad de usar tecnologías digitales, pero resulta más difícil hacerlo en un modelo de aula física con un profesor, 25 alumnos y tecnologías analógicas (Song et al., 2012). Un entorno híbrido ofrece múltiples recursos, vías diversificadas para alcanzar los objetivos de aprendizaje, distintos formatos de actividad conjunta y de interacción entre profesores y alumnos, y entre alumnos, recursos que no solo hacen más fácil el despliegue de las estrategias de personalización, sino que además permiten ampliar considerablemente su alcance y eficacia. Ahora bien, las posibilidades que ofrecen las TIC para apoyar las estrategias de personalización del aprendizaje no dependen tanto de las características de la tecnología o tecnologías seleccionadas como del uso que se hace de ellas en el diseño y desarrollo de las actividades de enseñanza y aprendizaje que las incorporan (Coll et al., 2008). De ahí que, cuando nos proponemos hibridar un entorno de enseñanza y aprendizaje, haya que empezar preguntándose sobre el valor añadido que las TIC pueden aportar a los procesos de aprendizaje que pretendemos impulsar. En los entornos híbridos orientados a la personalización del aprendizaje, las TIC pueden y deben servir para facilitar, transformar o potenciar las estrategias de personalización, no para reproducirlas sin más.

En lo que sigue presentamos e ilustramos con algunos ejemplos cómo la configuración de entornos híbridos de enseñanza y aprendizaje permite integrar de forma muy flexible periodos en los que profesores y alumnos trabajan "alrededor" de los dispositivos digitales con otros en los que trabajan "a través" de los dispositivos digitales (Crook, 1994), borrando así las fronteras no solo entre los contextos 
presenciales y los virtuales, sino también entre los contextos escolares y los no escolares. Esta integración está en la base de la potencialidad de los entornos híbridos para ampliar el alcance y la eficacia de las estrategias de personalización, al tiempo que facilita que el profesorado pueda hacer un seguimiento y un acompañamiento de los procesos de aprendizaje del alumnado en su tránsito por los contextos de dentro y fuera de la escuela.

\section{i. El uso de las tecnologías digitales para conectar contextos de aprendizaje}

El diseño de actividades social y culturalmente relevantes para el alumnado es una estrategia básica para favorecer la realización de aprendizajes escolares con sentido y valor personal para el alumnado. Para ello, es necesario abrir la escuela a la colaboración con las instituciones de su entorno más próximo y ofrecer a las entidades ajenas a la educación formal presentes en el territorio la oportunidad de implicarse en la educación de los alumnos. Se trata de construir redes formadas por entidades culturales, sociales, científicas, etc., y poner todos los recursos de aprendizaje disponibles en la comunidad, incluidos los escolares, al servicio del aprendizaje de los alumnos. Un buen ejemplo de este tipo de iniciativas es el proyecto Educació 36o. Educació a temps complet (https://www.educacio360.cat) que se está llevando a cabo en Catalunya (España) y cuya finalidad es impulsar un cambio profundo en el sistema educativo catalán mediante la integración de los aprendizajes que se tienen lugar en los diferentes tiempos y espacios de la vida de los niños, adolescentes y jóvenes (Coll et al., 2020).

Las TIC pueden contribuir a difuminar las fronteras entre los distintos contextos de aprendizaje en que participan los alumnos, como se pretende en el proyecto Educació 360 y en otros proyectos con características similares, mediante el diseño de actividades auténticas (Díaz Barriga, 2006) que sean relevantes para el alumnado y que enlacen con problemas, intereses o necesidades de su comunidad (laborales, políticos, cívicos, éticos, etc.). Un primer uso de las TIC para apoyar la personalización del aprendizaje consiste en utilizar estas tecnologías para crear redes y establecer acuerdos de colaboración con los actores sociales y comunitarios presentes en los equipamientos culturales locales (museos, bibliotecas, asociaciones u otros servicios de la comunidad) para el desarrollo de proyectos educativos compartidos.

En el desarrollo de este tipo de proyectos, los dispositivos móviles y la amplia colección de aplicaciones (apps) que existen en la actualidad para los mismos permiten llevar los procesos de aprendizaje más allá del aula a otros espacios naturales, urbanos, culturales o sociales de la comunidad. Es el caso, por ejemplo, del uso del propio dispositivo móvil para involucrar a los alumnos en procesos de indagación científica en un entorno de aprendizaje que integra el aula, el hogar, el laboratorio de la escuela y el contexto en línea. Con el apoyo de diversas aplicaciones, la cámara y funciones de grabación los alumnos pueden recopilar datos del fenómeno 
objeto de estudio, buscar información, compartir sus hallazgos y reflexiones con sus compañeros y el profesorado, y reflexionar sobre el proceso y los resultados de la investigación (Song y Wen, 2018).

Una vez finalizados los proyectos, la difusión por Internet de los productos que los alumnos elaboran constituye un tercer tipo de uso de las TIC que facilita el establecimiento de conexiones entre los distintos contextos de aprendizaje y contribuye a reforzar el sentido y valor personal que los alumnos atribuyen a las actividades escolares. No se trata ya de realizar el trabajo para el profesorado, sino de mostrar lo aprendido a una audiencia real formada por otros agentes educativos, los pares, los familiares o la comunidad en general. En la actualidad prácticamente todos los centros educativos mantienen páginas web o blogs que permiten al alumnado publicar de forma ágil sus trabajos. O como en el proyecto \#Estamoscontigo del CEIP Alba Plata que utilizan YouTube para publicar las cartas de ánimo a los "héroes y heroínas que estaban luchando frente a la pandemia" que han elaborado los alumnos durante la pandemia (García y Durán, 2021).

\section{ii. El uso de las tecnologías digitales para conectar experiencias de aprendizaje}

Uno de los objetivos de la personalización del aprendizaje es ayudar al alumnado a construir trayectorias personales de aprendizaje estableciendo relaciones entre las experiencias de aprendizaje y los aprendizajes que realiza en los diferentes contextos, escolares y no escolares, por los que transita. Desde el punto de vista del aprendiz, el aspecto esencial no son tanto las conexiones entre los contextos como entre las experiencias de aprendizaje y los aprendizajes que tienen lugar en esos contextos (Coll, 2016; Engel y Membrive, 2018).

Situar las trayectorias personales como foco de la acción educativa implica favorecer el acceso de todo el alumnado a los contextos de actividad que ofrecen mejores oportunidades, recursos y herramientas para aprender. Un buen ejemplo del papel que pueden jugar las TIC en este sentido es Cities of Learning (https:// www.home.lrng.org), una iniciativa comunitaria que ayuda a conectar a los jóvenes con una amplia gama de oportunidades de aprendizaje en sus ciudades en función de sus intereses. Este proyecto pone a disposición de los jóvenes y sus familias en una única plataforma en línea las actividades de aprendizaje, en línea y presenciales, de cientos de organizaciones comunitarias. Por ejemplo, en Chicago mediante Cities of Learning, 120 organizaciones han brindado un total de 4.500 oportunidades de aprendizaje. A medida que los alumnos participan en estas actividades de aprendizaje, obtienen insignias digitales en función de sus logros. Estas insignias digitales acreditan el dominio de una habilidad como, por ejemplo, codificación, juegos digitales, diseño o moda, etc., dando así mayor visibilidad al aprendizaje y documentándolo dondequiera que ocurra. Debido a que las insignias digitales contienen información detallada sobre las experiencias de aprendizaje de cada 
aprendiz, el profesorado de los centros educativos a los que asisten estos jóvenes puede tener una visión más completa de sus intereses y competencias, lo que permite ayudarles a tender puentes entre esas experiencias de aprendizaje y apoyarles en la construcción de su trayectoria personal de aprendizaje.

Por otra parte, los dispositivos inalámbricos, móviles y con conexión permanente a Internet ofrecen la posibilidad de cambiar de contexto de actividad con facilidad y rapidez, y también de llevar con uno mismo los instrumentos de aprendizaje al transitar de un contexto a otro. Estas posibilidades permiten diseñar actividades favorecedoras de que las habilidades o competencias aprendidas en el aula puedan ser analizadas, enriquecidas, reutilizadas y/o "remezcladas" posteriormente en otros espacios de aprendizaje fuera de la escuela; y a la inversa, que lo aprendido en otros espacios de aprendizaje pueda ser resignificado en las aulas. Se trata, en suma, de que tanto las competencias y los contenidos aprendidos como el proceso mismo de aprendizaje estén permanentemente abiertos a una "recontextualización" asociada a la vivencia de continuidad de los aprendizajes, a "un aprendizaje sin costuras" -Seamless Learning- (Wong y Looi, 2011), con independencia del contexto y del momento en que han tenido lugar.

La toma en consideración de todas las experiencias de aprendizaje de los alumnos, y no solo de aquellas que tienen lugar en el contexto de educación formal escolar, es un aspecto central de una acción educativa orientada a promover y favorecer que los alumnos puedan relacionar los contenidos escolares de aprendizaje con sus intereses, motivos y opciones personales; es decir, de una acción educativa que aspira a promover y reforzar el sentido y valor personal que el alumnado atribuye a las actividades escolares y, con ello, su implicación en el aprendizaje.

\section{iii. El uso de las tecnologías digitales para conectar el aprendizaje escolar con los intereses de los alumnos}

Tomar en consideración los intereses y objetivos del alumnado en el diseño, la planificación y desarrollo de las actividades de enseñanza y aprendizaje no significa presentar a los alumnos y alumnas un menú de temáticas para que elijan cuáles quieren trabajar y aprender y cómo quieren hacerlo. Por el contrario, desde esta perspectiva de la personalización del aprendizaje, trabajar con y a partir de los intereses significa diseñar actividades y situaciones que permitan ayudar al alumnado a identificar sus intereses, a contrastarlos con los intereses de sus pares, a valorarlos, a reflexionar sobre ellos, a resignificarlos, a generar nuevos intereses y a convertirlos en el objetivo que guía su aprendizaje. El interés no es algo que proceda de una disposición personal, ni que se genere en el vacío. Los intereses surgen en el marco de las actividades y de las prácticas socioculturales en las que los alumnos participan con otros, en el seno de las familias, de las comunidades de pertenencia y en la escuela (Renninger y Hidi, 2016). Trabajar con y a partir de los intereses de los alumnos en la escuela significa hacerlos compatibles con los objetivos de 
aprendizaje, ayudar al alumnado a desarrollar intereses en torno a los contenidos escolares y darles apoyo para que los concreten y persigan más allá de la escuela (Coll 2018; Engel et al., 2021; Solari, 2020).

En lo que sigue vamos a comentar brevemente tres tipos de usos de las tecnologías digitales que permiten apoyar, mediante herramientas específicas, distintos aspectos implicados en el trabajo con y a partir de los intereses del alumnado y vincular no solo los intereses que los alumnos generan fuera de la escuela con los contenidos escolares, sino también ayudarles a perseguir fuera de la escuela los intereses generados en la escuela: la presentación de los propios intereses, el contraste con los de otros y la ampliación o profundización de los mismos.

El primer tipo de uso tiene que ver con la identificación, presentación y representación de los intereses. En la actualidad disponemos de un amplio conjunto de herramientas digitales que permiten que el alumnado represente visualmente sus intereses a partir de nodos que pueden simbolizar objetos, acontecimientos, contenidos, prácticas socioculturales u otros tipos de informaciones, que pueden unirse mediante vínculos para formar redes no lineales de nodos (por ejemplo: Mindmeister, https://www.mindmeister.com; Bubbl, https://bubbl.us; Coggle, https://coggle.it). Estas herramientas permiten, por lo general, utilizar textos, imágenes e iconos, e incluso sonidos, animaciones e hipervínculos a informaciones externas. La mayoría de ellas ofrecen además librerías de recursos que ayudan a elaborar representaciones con un diseño muy atractivo. Construir una representación visual de los propios intereses mediante este tipo de herramientas es un proceso creativo, consciente e intencional que puede ayudar a los alumnos a dialogar consigo mismos y cuestionarse lo que les interesa aprender. Los alumnos pueden elaborar a lo largo del curso sucesivas representaciones de sus intereses, y contrastar cada nueva versión con las anteriores para analizar los que se mantienen, pierden importancia o se consolidan a medida que desarrollan proyectos y actividades a lo largo del curso. La mayor parte de estas herramientas permiten también que los alumnos construyan representaciones grupales de sus intereses, propiciando el diálogo y la reflexión conjunta sobre los mismos.

Un segundo uso de las tecnologías para el trabajo con y a partir de los intereses son los foros, que pueden configurarse como espacios propicios para que los alumnos presenten y contrasten sus intereses de aprendizaje con sus pares y el docente. El tipo de comunicación propia de los foros - asíncrona, multidireccional y basada en los textos escritos, aunque permite también adjuntar figuras, gráficos o vídeosfavorece que los alumnos puedan expresar con mayor precisión los significados que quieren transmitir y preparar respuestas argumentadas. La permanencia del texto escrito y de los archivos adjuntos hace posible, además, que puedan revisar y analizar de forma reflexiva las ideas presentadas por ellos mismos y por los otros.

Una tercera manera de promover el trabajo con los intereses de los alumnos mediante las tecnologías digitales consiste en usarlas para ampliar las fuentes de información presentes en las clases. Actualmente existen repositorios de 
recursos educativos abiertos y gratuitos sobre prácticamente cualquier ámbito de conocimiento que utilizan lenguajes y formatos diversos -lenguaje escrito, lenguaje oral, vídeos, representaciones gráficas, simulaciones, animaciones, etc.-, a menudo en combinación, y que facilitan que los alumnos amplíen y profundicen en sus intereses de aprendizaje, o incluso que construyan nuevos intereses. En general, este tipo de recursos han sido elaborados de forma que pueden ser modificados y adaptados con relativa facilidad a distintos contextos de uso, tanto en el aula como en el hogar u otros contextos de actividad (por ejemplo: Khan Academy https:// es.khanacademy.org; TED-Ed, Lessons https://ed.ted.com/lessons; Ck-12 https:// www.ck12.org; Procomún https://procomun.educalab.es).

\section{iv. El uso de las tecnologías digitales para facilitar el seguimiento del alumnado y coordinación del profesorado}

La personalización del aprendizaje implica, como ya se puede haber intuido a partir de los ejemplos presentados, tiempo y recursos para un seguimiento más profundo y sistemático del alumnado de lo que exigen planteamientos pedagógicos de tipo más tradicional. El seguimiento sistemático de los itinerarios que hace el alumnado a través de distintos espacios de aprendizaje y de los aprendizajes que en ellos realiza exige, a nuestro juicio, reforzar al menos dos aspectos clave estrechamente relacionados: la tutorización y atención personalizada al alumnado y la coordinación del profesorado.

El seguimiento, acompañamiento y soporte al alumnado es crucial en propuestas educativas que dan a los alumnos la oportunidad de recorrer distintos espacios de aprendizaje; cuanto más se diversifican esos espacios, mayor es la necesidad de una tutorización intensiva. Es importante que los equipos docentes conozcan en profundidad a cada alumno, sus intereses, fortalezas y debilidades, y sus proyectos de futuro, así como su contexto familiar y social, para poder personalizar su aprendizaje. Este seguimiento y la atención continuada se puede realizar tanto de forma presencial como en línea, de forma individual y en grupo, y también con la familia. El seguimiento sistemático de los itinerarios que realiza del alumnado a través de distintos espacios de aprendizaje y de los aprendizajes que en ellos realiza exige reforzar la coordinación entre los docentes del centro para compartir sus progresos y dificultades en el proceso de aprendizaje y para asegurarse de que recibe las ayudas educativas que necesita en cada momento. Lo deseable es que esta coordinación se amplíe para implicar también a los agentes educativos presentes en los distintos espacios en los que aprende el alumnado.

Las tecnologías digitales pueden convertirse en un buen aliado para apoyar la tutorización sistemática del alumnado y la coordinación del profesorado mediante el registro del tránsito del alumnado por los diferentes espacios y actividades del centro y de los aprendizajes alcanzados en ellos. Poner en marcha una estructura y procedimiento de registro en línea común a todo el centro comporta un importante 
trabajo previo del profesorado dirigido a acordar y compartir como mínimo tres decisiones: i) qué evidencias de aprendizaje es más relevante recoger y registrar en cada uno de los espacios de aprendizaje y en qué momentos es recomendable hacerlo; ii) la identificación de las competencias que se pretende que el alumnado trabaje en los diferentes espacios de aprendizaje; y iii) una nomenclatura común para evaluar y valorar el grado de desempeño alcanzado por el alumnado en las competencias identificadas como objeto de aprendizaje en cada espacio. Este tipo de registro, al que debería poder acceder todo el profesorado implicado en los espacios de aprendizaje por los que transitan los alumnos, debería recoger las valoraciones del equipo docente, así como las anotaciones de los profesores relativas a sus alumnos tutorados y, en su caso, propuestas de actuaciones para reforzar, ampliar o profundizar determinados aprendizajes.

Reigeluth y colaboradores (Reigeluth, 2017) han propuesto un sistema denominado Personalized Integrated Educational System (PIES) con cuatro funciones o espacios principales para apoyar procesos de personalización del aprendizaje: i) el registro de los datos del alumno, académicos y no académicos como intereses, características personales u objetivos profesionales; ii) el plan personal de aprendizaje de cada alumno; iii) las actividades de aprendizaje del alumnado y los recursos e instrumentos necesarios para su ejecución o desarrollo (simulaciones, tutoriales, ejercicios mecánicos y de práctica, etc.), y herramientas para monitorizar los progresos en el aprendizaje; y iv) las actividades e instrumentos de evaluación formativa y sumativa, y de retroalimentación al alumnado. En el sistema los datos de las cuatro funciones están integradas, de modo que la información es compartida por todos los usuarios (estudiantes, familias, profesorado).

Dos ejemplos comerciales de este tipo de tecnología son Additio (https:// www.additioapp.com) y iEduca (https://www.ieduca.com/es). Estas plataformas permiten al profesorado compartir información sobre el desempeño del alumnado y cuentan, además, con aplicaciones mediante las cuales las familias pueden hacer el seguimiento y acompañamiento de sus hijos en la escuela. También contemplan la posibilidad de que el profesorado de las escuelas y los institutos se coordine con agentes educativos que operan fuera del contexto escolar. Son, por lo demás, plataformas que pueden integrarse en Moodle o el sistema Google for Education e incluyen funciones no solo de gestión académica del centro sino también de gestión administrativa.

\section{CONSIDERACIONES FINALES: HACIA LA CONSTRUCCIÓN DE ENTORNOS PERSONALES DE APRENDIZAJE HÍBRIDOS}

Como ya se ha mencionado, la articulación de distintas estrategias de personalización del aprendizaje en una misma práctica educativa o espacio de aprendizaje incrementa las posibilidades de asegurar que el alumnado atribuya un elevado sentido y valor personal a los aprendizajes (Coll, 2018). En esta línea, 
queremos cerrar el presente trabajo llamando la atención sobre las posibilidades que los entornos personales de aprendizaje (Personal Learning Environment; PLE, por sus iniciales en inglés) ofrecen para generar prácticas educativas o espacios de aprendizaje capaces de integrar las distintas estrategias de personalización del aprendizaje que hemos comentado en las páginas precedentes.

Los entornos educativos personalizados ponen el énfasis en la toma en consideración de la voz del alumno y el reconocimiento y aceptación de su capacidad de decisión en la dirección, control y responsabilidad de su propio proceso de aprendizaje. Cuando un aprendiz diseña su propio PLE, tiene que tomar una serie de decisiones que implican incorporar y organizar, en función de sus intereses, objetivos y opciones personales, sus propios recursos, herramientas y aplicaciones para aprender, que no tienen por qué ser exclusivamente digitales ni exclusivamente individuales. Por el contrario, los PLE pueden ser híbridos, y a menudo lo son, en el sentido de que incluyen recursos y herramientas no digitales como libros en papel, escritorios, lápices y folios... que son utilizados para aprender. Igualmente, los PLE incorporan la red de personas (pares, profesorado, mentores, empleadores, etc.) con quien el aprendiz interactúa y se involucra en actividades de aprendizaje compartidas. Cada aprendiz configura su PLE de manera diferente en función de sus objetivos e intereses de aprendizaje, así como en función de las necesidades y oportunidades de aprendizaje que caracterizan el ciclo vital en que se encuentra (Castañeda et al., 2019; Coll y Engel, 2014; Dabbagh y Castañeda, 2020).

Para algunos autores (Attwell, 2019) uno de los aspectos más prometedores de los PLE es la posibilidad que ofrecen al aprendiz de conectar e integrar las experiencias de aprendizaje formales, no formales e informales, con independencia del momento y lugar en que han tenido lugar, facilitando el aprendizaje permanente o continuo. En ese sentido, el aprendiz se convierte en el diseñador de un espacio de aprendizaje que le permite construirse itinerarios educativos significativos y flexibles adaptados a los objetivos o intereses de aprendizaje que se plantea en cada momento de su vida traspasando los límites institucionales y organizacionales. Así, un interés de aprendizaje generado en la escuela puede ser satisfecho o ampliado por el aprendiz en otros tipos de contextos de actividad como una comunidad virtual de práctica o una asociación cultural de su población.

Desde la perspectiva de la personalización del aprendizaje, los PLE pueden ser un importante recurso para favorecer la reflexión del alumnado sobre las experiencias de aprendizaje y los aprendizajes que realiza en los diferentes contextos, escolares y no escolares, en los que participa y ayudarle a construirse una trayectoria personal de aprendizaje cada vez más potente y habilitadora. Igualmente pueden ser recursos para orientar la reflexión de los alumnos sobre sus propias características como aprendices, sus objetivos e intereses de aprendizaje, sus fortalezas y debilidades, las emociones asociadas a las distintas experiencias de aprendizaje, su capacidad para crear situaciones y condiciones que les facilitan el aprendizaje, las estrategias que ponen en marcha para afrontar las dificultades o contratiempos, los recursos 
que les han permitido superarlas y las fuentes de ayuda a las que han recurrido. La incorporación a los PLE de herramientas con funcionalidades similares a las de los portafolios (Barberà y de Martín, 2011) permitiría, además, incrementar su utilidad para apoyar a los alumnos en los procesos reflexivos, al tiempo que facilitaría el seguimiento y acompañamiento de los aprendizajes entre los distintos contextos de actividad por los que transitan. En suma, los PLE, entendidos como entornos híbridos de aprendizaje, ilustran perfectamente el valor añadido de las tecnologías digitales de la información y la comunicación, o para ser más precisos, de determinados usos de estas tecnologías, para personalizar el aprendizaje.

\section{NOTA}

1. Este trabajo ha sido realizado como parte de un proyecto de investigación financiado por el Ministerio de Economía, Industria y Competitividad del gobierno de España (referencia EDU2017-82321-R). Se puede obtener más información sobre este proyecto y el grupo de investigación en https://ble.psyed.edu.es

\section{REFERENCIAS}

Attwell, G. (2019). E-learning at the workplace. En S. McGrath, M. Mulder, J. Papier, y R. Suart (Eds.), Handbook of vocational education and training: developments in the changing world of work (pp. 1-25). Springer. https://doi. org/10.1007/978-3-319-49789-1 110-1

Barberà, E., y de Martín, E. (2011). Portfolio electrónico: aprender a evaluar el aprendizaje. Editorial UOC.

Bray, B., y McClaskey, K. (2014). Make learning personal: The what, who, wow, where, and why. Corwin Press.

Castañeda, L., Tur, G., y Torres-Kompen, R. (2019). Impacto del concepto PLE en la literatura sobre educación: la última década. RIED. Revista Iberoamericana de Educación a Distancia, 22(1), 221-241. https://doi.org/10.5944/ried.22.1.22079

Coll, C. (2016). La personalització de l'aprenentatge escolar. El què, el per què i el com d'un repte indefugible. En A J. M. Vilalta (Dr.), Reptes de l'educació a Catalunya. Anuari d'Educació 2015 (pp. 43-104). Fundació Bofill.
Coll, C. (2018). Personalización del aprendizaje. Editorial Graó.

Coll, C., y Engel, A. (2014). Los entornos personales de aprendizaje en contextos de educación formal. Cultura y Educación, 26(4), 617-630. https://doi.org/10.1080/ 11356405.2014 .985947

Coll, C., Esteban-Guitart, M., e Iglesias, E. (2020). Aprenentatge amb sentit $i$ valor personal. Estratègies, recursos $i$ experiències de personalització educativa. Ed. Graó

Coll, C., Mauri, T., y Onrubia, J. (2008). La utilización de las tecnologías de la información y la comunicación en la educación: Del diseño tecno-pedagógico a las prácticas de uso. En C. Coll y C. Monereo (Eds.), Psicología de la educación virtual. Enseñar y aprender con las tecnologías de la información y la comunicación (pp. 74-103). Ed. Morata.

Consejo Escolar del Estado (2021). Situación actual de la educación en España a consecuencia de la pandemia. https:// www.educacionyfp.gob.es/mc/cee/ 
publicaciones/estudios/situacionpandemia.html

Crook, C. (1994). Computers and the collaborative experience of learning. Routledge.

Cuban, L. (2018). The Flight of a Butterfly or the Path of a Bullet? Using Technology to Transform Teaching and Learning. Harvard Education Press.

Dabbagh, N., y Castañeda, L. (2020). The PLE as a framework for developing agency in lifelong learning. Education Tech Research Dev, 68, 3041-3055. https:// doi.org/10.1007/s11423-020-09831-Z

Departament d'Educació i Institut de Recerca en Educació, Universitat de Barcelona (2021). Informe projecte pilot d'Educació Híbrida.

Díaz Barriga, F. (2006). Enseñanza situada: vínculo entre la escuela y la vida. McGraw-Hill.

Dumont, H. D., Instance, D., y Benavides, F. (Eds.) (2010). The nature of Learning. Using Research to Inspire Practice. OECD. https://doi. org/10.1787/9789264086487-en

Engel, A., y Membrive, A. (2018). Contextos de actividad, experiencias de aprendizaje y trayectorias personales. En C. Coll (Coord.), Personalización del aprendizaje (pp. 19-22). Ed. Graó.

Engel, A., Vizquerra, M. I., y Solari, M. (2021). Trabajar con y a partir de los intereses de aprendizaje del alumnado. Página web Cuadernos de Pedagogía. http://www.is.gd/tVTbXH

Fundación SM (2020). Volvemos a clase. El impacto del confinamiento en la educación. https://www.fundacion-sm. org/investigacion/informe-volvemos-aclase

García, R. J., y Durán, J. A. (2021). Estamos contigo: Escuelas en red. El País https:// elpais.com/elpais/2021/03/10/escuelas en red/1615375969 148158.html

Hodges, C. B., Moore, S., Lockee, B. B., Trust, T., y Bond, M. A. (2020). The difference between emergency remote teaching and online learning. Educause Review, March 27, 2020 https://er.educause.edu/ articles/2020/3/the-difference-betweenemergency-remote-teaching-and-onlinelearning

Learning Policy Institute \& Turnaround for Children. (2021). Design principles for schools: Putting the science of learning and development into action. https:// learningpolicyinstitute.org/sites/default/ files/product-files/SoLD Design Principles REPORT.pdf

Miao, F., Huang, R., Liu, D., y Zhuang, R. (2020). Ensuring effective distance learning during COVID-19 disruption: guidance for teachers (Spanish). UNESCO https://unesdoc.unesco.org/ ark:/48223/pfooo0375116spa

Reigeluth, Ch. M. (2017). Designing Technology for the Learner-centered Paradigm of Education. En Ch. Reigeluth, B. J. Beatty y R. D. Myers (Eds.) (2017). Instructional Design Theories and Models. The LearnerCentered Paradigm of Education. Vol. $I V$ (pp. 287-316). Routledge. https://doi. org/10.4324/9781315795478

Renninger, K. A., y Hidi, S. (2016). The power of interest for motivation and engagement. Routledge. https://doi. org/10.4324/9781315771045

Sawyer, R. K. (Ed.) (2014). The Cambridge Handbook of The Learning Sciences. Second Edition. Cambridge University Press. $\quad$ https://doi.org/10.1017/ CBO9781139519526

Solari, M. (2020). Una aproximación teórico-metodológica a los intereses del alumnado como recurso para la construcción de aprendizajes con sentido. Conferencia en el Posgrado de Psicología de la Educación. Barcelona, 5 de noviembre. https://youtu.be/ f52zXopnJyE

Song, Y., y Wen, Y. (2018). Integrating various apps on BYOD into seamless 
inquiry-based learning to enhance primary students' science learning. Journal of Science Education and Technology, 27(2), 165-176. https://doi. org/10.1007/s10956-017-9715-Z

Song, Y., Wong, L. H., y Looi, C. K. (2012). Fostering personalized learning in science inquiry supported by mobile technologies. Educational Technology Research and Development, 6o(4), 679-701. https:// doi.org/10.1007/s11423-012-9245-6

Toshalis, E., y Nakkula, M. J. (2012). Motivation, engagement, and student voice: The student at the center series. Jobs for the Future and Nellie Mae Foundation. https://www. howyouthlearn.org/pdf/Motivation\%20 Engagement\%20Student\%20Voiceo.pdf

UNICEF (2020). Encuesta para conocer la opinión del personal docente. https:// www.unicef.es/educa/blog/docentesfrente-al-coronavirus

Department of Education (2016). Future Ready Learning: Reimagining the Role of Technology in Education. National Education Technology Plan. Office of Educational Technology. http://tech. ed.gov

Wong, L. H., y Looi, C. K. (2011). What seams do we remove in mobile-assisted seamless learning? A critical review of the literature. Computers and Education, 57, 2364-2381. https://doi.org/10.1016/j. compedu.2011.06.007

WorldBank(2020).GuidanceNoteonRemote Learning and COVID-19. World Bank Group. http://documents.worldbank. org/curated/en/344621586550566257/ Guidance-Note-on-Remote-Learningand-COVID-19

\section{PERFIL ACADÉMICO Y PROFESIONAL DE LOS AUTORES}

Anna Engel. Doctora en Psicología y profesora agregada de la Universidad de Barcelona. Miembro del Grupo de Investigación en Interacción e Influencia Educativa (GRINTIE) y coordinadora general del Máster Interuniversitario de Psicología de la Educación. https://orcid.org/oooo-0003-1153-2101

E-mail: anna.engel@ub.edu

César Coll. Doctor en Psicología y catedrático emérito de Psicología Evolutiva y de la Educación de la Universidad de Barcelona. Coordinador del Grupo de Investigación en Interacción e Influencia Educativa (GRINTIE). https://orcid. org/o000-0001-7669-6268

E-mail: ccoll@ub.edu

\section{DIRECCIÓN DE LOS AUTORES}

Universitat de Barcelona.

Campus Mundet, Ponent.

Passeig de la Vall d'Hebron, 171

o8035 Barcelona, España 
Fecha de recepción del artículo: 09/09/2021

Fecha de aceptación del artículo: 14/09/2021

Fecha de aprobación para maquetación: 08/10/2021 